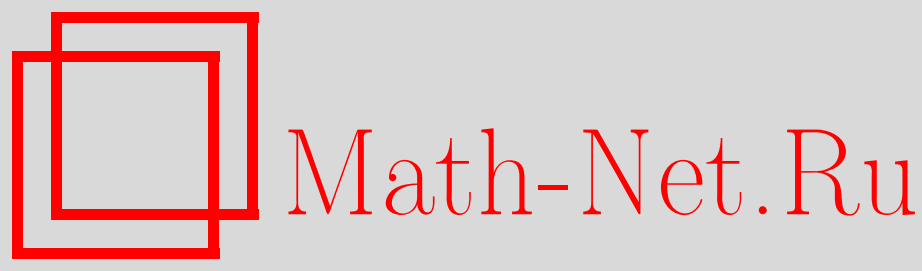

А. Али, К. Хамброк, А. Я. Пархоменко, Волновые функции тяжелых барионов вблизи светового конуса, ТМФ, 2012, том 170, номер 1, 5-21

DOI: https://doi.org/10.4213/tmf6742

Использование Общероссийского математического портала Math-Net.Ru подразумевает, что вы прочитали и согласны с пользовательским соглашением http: //www . mathnet.ru/rus/agreement

Параметры загрузки:

IP: 34.239 .49 .27

26 апреля 2023 г., $11: 48: 42$

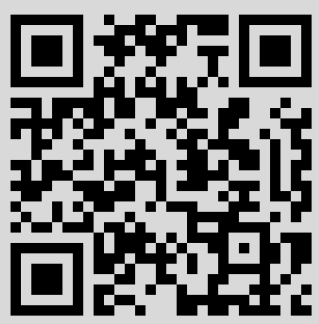




\title{
ВОЛНОВЫЕ ФУНКЦИИ ТЯЖЕЛЫХ БАРИОНОВ ВБЛИЗИ СВЕТОВОГО КОНУСА
}

\begin{abstract}
Представлена классификация трехкварковых амплитуд распределения, определенных на световом конусе, для тяжелых барионов в основном состоянии со спин-четностью $J^{P}=1 / 2^{+}$и $J^{P}=3 / 2^{+}$в рамках квантовой хромодинамики в пределе симметрии тяжелых кварков. Вычислены несколько первых моментов для амплитуд распределения методом правил сумм квантовой хромодинамики. Предложены простые модельные функции для амплитуд распределения тяжелых барионов, и проанализирована их зависимость от энергетического масштаба.
\end{abstract}

Ключевые слова: тяжелые барионы, амплитуда распределения, правила сумм.

\section{1. ВВЕДЕНИЕ}

За практически десятилетний период своей работы фабрики $B$-мезонов в SLAC и KEK внесли значительный вклад в прояснение природы $C P$-нарушения в кварковом секторе стандартной модели. Изучение физики тяжелых барионов, содержащих $b$-кварк, на LHC может служить дополнительным тестом для проверки механизма Кобаяши и Маскава. Специфичные процессы с участием $b$-барионов такие, как редкие распады, обусловленные нейтральными токами с изменением аромата (НТИА), рассматриваются в качестве потенциальных источников для обнаружения новой физики за рамками стандартной модели. В отличие от $B$-мезонов наличие ненулевого спина у барионов допускает также экспериментальное изучение корреляций спинов. За последние несколько лет спектр тяжелых $b$-барионов существенно расширился благодаря достижениям коллабораций CDF и D0 с ускорителя Tevatron. Несмотря на этот прогресс, изучение распадов тяжелых барионов, идущих за счет НТИА, ограничено статистикой событий. Больших успехов следует ожидать от LHC, на котором $b$-барионы будут рождаться в изобилии, и их слабые распады могут быть измерены с точностью, достаточной для выявления физики за рамками стандартной модели.

* Deutsches Elektronen-Synchrotron DESY, Notkestrasse 85, 22603 Hamburg, Germany. E-mail: ahmed.ali@desy.de, hambrock@mail.desy.de

† Ярославский государственный университет им. П.Г. Демидова, Ярославль, Россия. E-mail: parkh@uniyar.ac.ru 
Теория распадов $b$-барионов на легкие адроны намного сложнее распадов $B$-мезонов и, следовательно, менее востребована. Вычисления распадов тяжелых барионов на легкие частицы с использованием разложения по массе тяжелого кварка (см., например, работу [1]) или с применением метода правил сумм квантовой хромодинамики (KХД), развитых в работах [2]-[4], требуют информации об элементарных непертурбативных объектах - амплитудах распределения (AР) тяжелых барионов. В течение длительного времени существующие модели для АР тяжелых барионов [5], [6] были основаны на кварковой модели и не полностью согласованы с КХД. В работе [7] представлена полная классификация трехкварковых амплитуд распределения вблизи светового конуса для $\Lambda_{b}$-бариона в рамках КХД в пределе симметрии тяжелых кварков, а также проанализирована зависимость от энергетического масштаба у АР главного твиста. В дополнение были предложены простые модели для АР, и их параметры были вычислены на основе оценок нескольких первых моментов методом правил сумм КХД. Анализ, проведенный в работе [7], может быть применен ко всем $b$-барионам, находящимся в основном состоянии, со спин-четностями $J^{P}=1 / 2^{+}$и $J^{P}=3 / 2^{+}$. Главные этапы и основные результаты анализа собраны в настоящей работе, а детали вычислений будут представлены в отдельной работе [8].

\section{2. ИНТЕРПОЛЯЦИОННЫЕ ТОКИ ТЯЖЕЛЫХ БАРИОНОВ}

В рамках эффективной теории тяжелых кварков (ЭТТК) барионы, содержащие один тяжелый кварк $Q=c, b$, классифицируются в соответствии с угловым моментом $\ell$ и пространственной четностью $p$ пары легких кварков, называемой дикварком (обсуждение этого вопроса изложено в монографии [9]). Тяжелые кварки - нерелятивистские частицы, которые в главном порядке разложения по $1 / m_{Q}$ не взаимодействуют с дикварком.

Барионы в основном состоянии $(\ell=0)$ со спин-четностью $J^{P}$ характеризуются спин-четностью $j^{p}$ дикварка. Из спинов легких кварков можно образовать два состояния с $j^{p}=0^{+}$и $j^{p}=1^{+}$. В состоянии с $j^{p}=0^{+}$спиновая часть волновой функции антисимметрична, поэтому из статистики Ферми и антисимметрии по цвету следует антисимметрия волновой функции по аромату. В результате получается барионное состояние с изоспином $I=0$, построенное из легких $u$ - и $d$-кварков, которое называют $\Lambda_{Q}$-барионом (спин-четность $J^{P}=1 / 2^{+}$). Если спин-четность дикварка $j^{p}=1^{+}$, то спиновая часть барионной волновой функции симметрична, а значит, симметрична и составляющая волновой функции в пространстве ароматов. Если ограничиться только легкими $u$ - и $d$-кварками, то можно построить два вырожденных состояния с изоспином $I=1$, которые называют $\Sigma_{Q^{-}}$и $\Sigma_{Q}^{*}$-барионами, имеющими спин-четности $J^{P}=1 / 2^{+}$и $J^{P}=3 / 2^{+}$соответственно. Учет $s$-кварка приводит к увеличению числа тяжелых барионов в мультиплете, который помимо изоспина будет характеризоваться "странностью" $S$. При $S=-1$ имеются два барионных состояния $\Xi_{Q}$ и $\Xi_{Q}^{\prime}$ с $J^{P}=1 / 2^{+}$, а также $\Xi_{Q}^{*}$-барион с $J^{P}=3 / 2^{+}$.

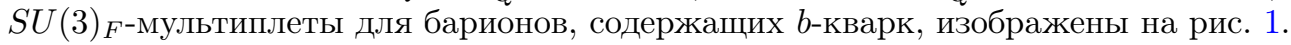

Локальные токи тяжелых барионов имеют следующий общий вид [10], [11]:

$$
J_{1}^{H_{Q}}=\varepsilon_{a b c}\left[\psi^{a \mathrm{~T}} \mathcal{C} \Gamma \mathcal{T} \psi^{b}\right] \Gamma^{\prime} Q_{v}^{c}, \quad J_{2}^{H_{Q}}=\varepsilon_{a b c}\left[\psi^{a \mathrm{~T}} \mathcal{C} \Gamma \not \mathcal{T} \psi^{b}\right] \Gamma^{\prime} Q_{v}^{c},
$$

где $a, b, c=1,2,3$ - цветовые индексы, $\psi-S U(3)_{F}$-триплет в пространстве ароматов, $v_{\mu}$ - четыре-скорость тяжелого кварка, $\not x=(v \gamma), Q_{v}$ - эффективное статическое поле тяжелого кварка, удовлетворяющее соотношению $\not Q_{v}=Q_{v}$, индекс Т означает 


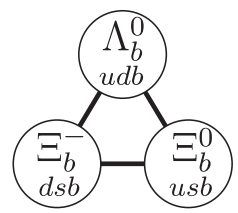

a

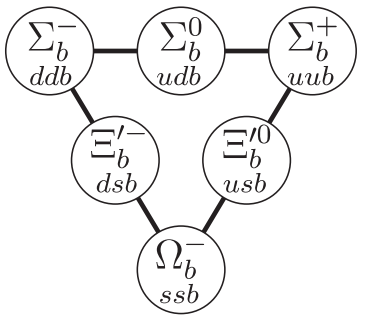

б

Рис. 1. Мультиплеты для $b$-барионов, реализуемые в соответствии с группой ароматов $S U(3)_{F}$ : а $-S U(3)_{F}$-триплет барионов со спин-четностью $J^{P}=1 / 2^{+}$, у которых легкие кварки образуют скалярное состояние $\left(j^{p}=0^{+}\right) ; \sigma-S U(3)_{F}$-сикстет барионов, у которых легкие кварки находятся в аксиально-векторном состоянии $\left(j^{p}=1^{+}\right)$. Состав частиц, представленный на этой диаграмме, соответствует $b$-барионам со спин-четностью $J^{P}=1 / 2^{+}$. Аналогичная диаграмма для барионов с $J^{P}=3 / 2^{+}$получается заменой всех названий частиц на идентичные с дополнительным верхним индексом $*$.

транспонирование, $\mathcal{C}$ - матрица зарядового сопряжения со свойствами $\mathcal{C} \gamma_{\mu}^{\mathrm{T}} \mathcal{C}^{-1}=$ $-\gamma_{\mu}$ и $\mathcal{C} \gamma_{5} \mathcal{C}^{-1}=\gamma_{5}$ и $\mathcal{T}-$ некоторая матрица в пространстве ароматов.

Для каждого бариона, находящегося в основном состоянии, имеются два независимых интерполяционных локальных тока $J_{1}$ и $J_{2}$ с подходящими квантовыми числами. Они могут быть построены в соответствии с правилами, предложенными в работах [10]-[13]. Для состояний из $S U(3)_{F}$-антитриплета $\overline{\mathbf{3}}$ (см. рис. 1а) эти токи имеют вид

$$
\begin{aligned}
J_{1}^{\Lambda_{Q}} & =\varepsilon_{a b c}\left[u^{a \mathrm{~T}} \mathcal{C} \gamma_{5} d^{b}\right] Q_{v}^{c}, & J_{2}^{\Lambda_{Q}} & =\varepsilon_{a b c}\left[u^{a \mathrm{~T}} \mathcal{C} \gamma_{5} \not b d^{b}\right] Q_{v}^{c}, \\
J_{1}^{\Xi_{Q}} & =\varepsilon_{a b c}\left[q^{a \mathrm{~T}} \mathcal{C} \gamma_{5} s^{b}\right] Q_{v}^{c}, & J_{2}^{\Xi_{Q}} & =\varepsilon_{a b c}\left[q^{a \mathrm{~T}} \mathcal{C} \gamma_{5} \not b s^{b}\right] Q_{v}^{c},
\end{aligned}
$$

где $q=u$ или $d$ - один из кварков, входящих в изодублет. Также имеются два $S U(3)_{F}$ сикстета 6 (см. рис. 1б), которые отличаются друг от друга спином. Для барионов с $J^{P}=1 / 2^{+}$локальные интерполяционные токи следующие:

$$
\begin{array}{rlrl}
J_{1}^{\Sigma_{Q}} & =-\varepsilon_{a b c}\left[q_{1}^{a \mathrm{~T}} \mathcal{C} \gamma_{t \mu} q_{2}^{b}\right] \gamma_{t}^{\mu} \gamma_{5} Q_{v}^{c}, & J_{2}^{\Sigma_{Q}} & =-\varepsilon_{a b c}\left[q_{1}^{a \mathrm{~T}} \mathcal{C} \gamma_{t \mu} \not b q_{2}^{b}\right] \gamma_{t}^{\mu} \gamma_{5} Q_{v}^{c}, \\
J_{1}^{\Xi_{Q}^{\prime}}=-\varepsilon_{a b c}\left[q^{a \mathrm{~T}} \mathcal{C} \gamma_{t \mu} s^{b}\right] \gamma_{t}^{\mu} \gamma_{5} Q_{v}^{c}, & J_{2}^{\Xi_{Q}^{\prime}}=-\varepsilon_{a b c}\left[q^{a \mathrm{~T}} \mathcal{C} \gamma_{t \mu} \not s^{b}\right] \gamma_{t}^{\mu} \gamma_{5} Q_{v}^{c}, \\
J_{1}^{\Omega_{Q}}=-\varepsilon_{a b c}\left[s^{a \mathrm{~T}} \mathcal{C} \gamma_{t \mu} s^{b}\right] \gamma_{t}^{\mu} \gamma_{5} Q_{v}^{c}, & J_{2}^{\Omega_{Q}}=-\varepsilon_{a b c}\left[s^{a \mathrm{~T}} \mathcal{C} \gamma_{t \mu} \not b s^{b}\right] \gamma_{t}^{\mu} \gamma_{5} Q_{v}^{c},
\end{array}
$$

где $\gamma_{t}^{\mu}=\gamma^{\mu}-\not v^{\mu}$. Для барионов с $J^{P}=3 / 2^{+}$ароматовая структура точно такая же, как и для барионов с $J^{P}=1 / 2^{+}$, но дираковская структура при поле тяжелого кварка другая. Проиллюстрируем эти токи на примере $\Sigma_{Q}^{*}$-бариона:

$$
\begin{aligned}
& J_{1 \mu}^{\Sigma_{Q}^{*}}=\varepsilon_{a b c}\left[q_{1}^{a \mathrm{~T}} \mathcal{C} \gamma_{t}^{\nu} q_{2}^{\prime b}\right]\left(g_{\mu \nu}-\frac{1}{3} \gamma_{t \mu} \gamma_{t \nu}\right) Q_{v}^{c}, \\
& J_{2 \mu}^{\Sigma_{Q}^{*}}=\varepsilon_{a b c}\left[q_{1}^{a \mathrm{~T}} \mathcal{C} \gamma_{t}^{\nu} \not b q_{2}^{\prime b}\right]\left(g_{\mu \nu}-\frac{1}{3} \gamma_{t \mu} \gamma_{t \nu}\right) Q_{v}^{c} .
\end{aligned}
$$

Для этих токов справедливо условие $\gamma_{t}^{\mu} J_{i \mu}^{\Sigma_{Q}^{*}}=0, i=1,2$. 
Матричные элементы локальных операторов (2)-(7) определяют параметры связи барионов $f_{H_{Q}}^{(i)}$ :

$$
\begin{array}{ll}
J^{P}=1 / 2^{+}: & \left\langle 0\left|J_{i}^{H_{Q}}\right| H_{Q}(v)\right\rangle=f_{H_{Q}}^{(i)} u^{H_{Q}}(v), \\
J^{P}=3 / 2^{+}: & \left\langle 0\left|J_{i \mu}^{H_{Q}^{*}}\right| H_{Q}^{*}(v)\right\rangle=\frac{1}{\sqrt{3}} f_{H_{Q}^{*}}^{(i)} u_{\mu}^{H_{Q}^{*}}(v),
\end{array}
$$

где коэффициент в матричном элементе $\left\langle 0\left|J_{i \mu}^{H_{Q}^{*}}\right| H_{Q}^{*}(v)\right\rangle$ выбран так, что $f_{H_{Q}}^{(i)}=$ $f_{H_{Q}^{*}}^{(i)}$ в пределе симметрии тяжелых кварков. Дираковский спинор $u^{H_{Q}}(v)$ тяжелых барионов $H_{Q}$, нормированный условием $\bar{u}^{H_{Q}}(v) u^{H_{Q}}(v)=1$ в нерелятивистском пределе, удовлетворяет соотношению $\not u^{H_{Q}}(v)=u^{H_{Q}}(v)$. Для $H_{Q}^{*}$-барионов волновая функция записывается через вектор-спиноры Рарита-Швингера $u_{\mu}^{H_{Q}^{*}}(v)$, для которых справедливы следующие соотношения ${ }^{1)}: \not u_{\mu}^{H_{Q}^{*}}(v)=u_{\mu}^{H_{Q}^{*}}(v)$ и $v^{\mu} u_{\mu}^{H_{Q}^{*}}(v)=$ $\gamma^{\mu} u_{\mu}^{H_{Q}^{*}}(v)=0$.

Суммирование по поляризациям приводит к следующему результату [14]:

$$
\begin{aligned}
& \sum_{\lambda=1}^{2} u^{H_{Q}(\lambda)}(v) \bar{u}^{H_{Q}(\lambda)}(v)=\frac{1+\not}{2} \equiv P_{+}, \\
& \sum_{\lambda=1}^{4} u_{\mu}^{H_{Q}^{*}(\lambda)}(v) \bar{u}_{\nu}^{H_{Q}^{*}(\lambda)}(v)=P_{+}\left[-g_{\mu \nu}+v_{\mu} v_{\nu}+\frac{1}{3} \gamma_{t \mu} \gamma_{t \nu}\right] .
\end{aligned}
$$

Легко проверить нормировку этих объектов:

$$
\operatorname{Sp} \sum_{\lambda=1}^{2} u^{H_{Q}(\lambda)}(v) \bar{u}^{H_{Q}(\lambda)}(v)=2, \quad-g^{\mu \nu} \operatorname{Sp} \sum_{\lambda=1}^{4} u_{\mu}^{H_{Q}^{*}(\lambda)}(v) \bar{u}_{\nu}^{H_{Q}^{*}(\lambda)}(v)=4,
$$

совпадающую с числом независимых поляризационных состояний.

AР тяжелых барионов определяются посредством матричных элементов перехода бариона в вакуум для нелокальных операторов струнного типа с подходящими квантовыми числами, построенных из эффективного поля $Q_{v}^{c}(0)$ тяжелого кварка и двух полей $q_{i}^{a}, i=1,2$, легких кварков. Для $\Lambda_{Q}$-бариона полный набор трехкварковых операторов струнного типа, классифицированных по твисту дикварка, был представлен в недавней работе [7]. Эти токи могут быть легко обобщены на случай $\Xi_{Q}$-барионов заменой одного из легких $u$ - или $d$-кварков на $s$-кварк и в общем виде могут быть записаны следующим образом:

$$
\begin{aligned}
J_{2}\left(t_{1}, t_{2}\right) & =\varepsilon_{a b c}\left[q_{1}^{a \mathrm{~T}}\left(t_{1} n\right) \mathcal{C} \gamma_{5} \not h q_{2}^{b}\left(t_{2} n\right)\right] Q_{v}^{c}(0), \\
J_{3 s}\left(t_{1}, t_{2}\right) & =\varepsilon_{a b c}\left[q_{1}^{a \mathrm{~T}}\left(t_{1} n\right) \mathcal{C} \gamma_{5} q_{2}^{b}\left(t_{2} n\right)\right] Q_{v}^{c}(0), \\
J_{3 \sigma}\left(t_{1}, t_{2}\right) & =\frac{i}{2} \varepsilon_{a b c}\left[q_{1}^{a \mathrm{~T}}\left(t_{1} n\right) \mathcal{C} \gamma_{5}(\bar{n} \sigma n) q_{2}^{b}\left(t_{2} n\right)\right] Q_{v}^{c}(0), \\
J_{4}\left(t_{1}, t_{2}\right) & =\varepsilon_{a b c}\left[q_{1}^{a \mathrm{~T}}\left(t_{1} n\right) \mathcal{C} \gamma_{5} \not h q_{2}^{b}\left(t_{2} n\right)\right] Q_{v}^{c}(0),
\end{aligned}
$$

1) Методику построения спинового состояния барионов, находящихся в возбужденном состоянии, можно найти в работе [14]. 
где $(\bar{n} \sigma n)=\bar{n}^{\mu} \sigma_{\mu \nu} n^{\nu}$, а ароматы легких кварков $q=u, d, s$ различаются, $q_{1} \neq q_{2}$ (кварковый состав реальных тяжелых барионов приведен на рис. 1). Нижние индексы 2, 3, 4 определяют твист оператора дикварка, $n^{\mu}$ и $\bar{n}^{\mu}$ - два изотропных вектора, нормированных условием $(\bar{n} n)=2$, которые выбраны так, что $v^{\mu}=\left(n^{\mu}+\bar{n}^{\mu}\right) / 2$ и $(n v)=(\bar{n} v)=1$. Матричные элементы операторов (13) можно параметризовать в соответствии с результатами работы [7]:

$$
\begin{aligned}
\left\langle 0\left|J_{2}\left(t_{1}, t_{2}\right)\right| H_{Q}(v)\right\rangle & =f_{H_{Q}}^{(2)} \Psi_{2}\left(t_{1}, t_{2}\right) u^{H_{Q}}(v), \\
\left\langle 0\left|J_{3 s}\left(t_{1}, t_{2}\right)\right| H_{Q}(v)\right\rangle & =f_{H_{Q}}^{(1)} \Psi_{3 s}\left(t_{1}, t_{2}\right) u^{H_{Q}}(v), \\
\left\langle 0\left|J_{3 \sigma}\left(t_{1}, t_{2}\right)\right| H_{Q}(v)\right\rangle & =f_{H_{Q}}^{(1)} \Psi_{3 \sigma}\left(t_{1}, t_{2}\right) u^{H_{Q}}(v), \\
\left\langle 0\left|J_{4}\left(t_{1}, t_{2}\right)\right| H_{Q}(v)\right\rangle & =f_{H_{Q}}^{(2)} \Psi_{4}\left(t_{1}, t_{2}\right) u^{H_{Q}}(v) .
\end{aligned}
$$

Наиболее простой способ построения полного набора трехчастичных АР для барионов со спин-четностью дикварка $j^{p}=1^{+}-$не учитывать спин тяжелого кварка (обозначим такой безспиновый кварк как $\widetilde{Q}_{v}$ ) и определить АР для "аксиально-векторного бариона" $\widetilde{H}_{Q}(v, \eta)$, который в этом случае характеризуется вектором поляризации $\eta^{\mu}$, удовлетворяющим условию $(v \eta)=0$. Определения для АР заимствованы из анализа векторных мезонов вблизи светового конуса [15]. Разделим восемь интерполяционных токов на две группы в полной аналогии с кирально-четными и кирально-нечетными АР для векторных мезонов [15]. Первый набор следующий:

$$
\begin{aligned}
J_{2 V}\left(t_{1}, t_{2}\right) & =\epsilon_{a b c}\left[q_{1}^{a \mathrm{~T}}\left(t_{1} n\right) \mathcal{C} \not h q_{2}^{b}\left(t_{2} n\right)\right] \widetilde{Q}_{v}^{c}(0), \\
J_{4 V}\left(t_{1}, t_{2}\right) & =\epsilon_{a b c}\left[q_{1}^{a \mathrm{~T}}\left(t_{1} n\right) \mathcal{C} \not h q_{2}^{b}\left(t_{2} n\right)\right] \widetilde{Q}_{v}^{c}(0), \\
J_{3 V}^{\mu}\left(t_{1}, t_{2}\right) & =\epsilon_{a b c}\left[q_{1}^{a \mathrm{~T}}\left(t_{1} n\right) \mathcal{C} \gamma_{\perp}^{\mu} q_{2}^{b}\left(t_{2} n\right)\right] \widetilde{Q}_{v}^{c}(0), \\
J_{3 A}^{\mu}\left(t_{1}, t_{2}\right) & =\epsilon_{a b c} \varepsilon_{\perp}^{\mu \nu}\left[q_{1}^{a \mathrm{~T}}\left(t_{1} n\right) \mathcal{C} \gamma_{\perp \nu} \gamma_{5} q_{2}^{b}\left(t_{2} n\right)\right] \widetilde{Q}_{v}^{c}(0),
\end{aligned}
$$

где $\gamma_{\perp}^{\mu}=\gamma^{\mu}-\left(\not h \bar{n}^{\mu}+\not h n^{\mu}\right) / 2, \varepsilon_{\perp}^{\mu \nu}=(i / 2) \varepsilon^{\mu \nu \rho \sigma} n_{\rho} \bar{n}_{\sigma}$ - антисимметричный тензор, peaлизованный в плоскости, перпендикулярной световому конусу (выполняется условие $\left.\varepsilon_{\perp \mu \nu} \varepsilon_{\perp}^{\nu \mu}=2\right)$. Второй набор:

$$
\begin{aligned}
J_{2 T}^{\mu}\left(t_{1}, t_{2}\right) & =\epsilon_{a b c}\left[q_{1}^{a \mathrm{~T}}\left(t_{1} n\right) \mathcal{C} \gamma_{\perp}^{\mu} \not h q_{2}^{b}\left(t_{2} n\right)\right] \widetilde{Q}_{v}^{c}(0), \\
J_{4 T}^{\mu}\left(t_{1}, t_{2}\right) & =\epsilon_{a b c}\left[q_{1}^{a \mathrm{~T}}\left(t_{1} n\right) \mathcal{C} \gamma_{\perp}^{\mu} \not h q_{2}^{b}\left(t_{2} n\right)\right] \widetilde{Q}_{v}^{c}(0), \\
J_{3 T}\left(t_{1}, t_{2}\right) & =\frac{i}{2} \epsilon_{a b c}\left[q_{1}^{a \mathrm{~T}}\left(t_{1} n\right) \mathcal{C}(\bar{n} \sigma n) q_{2}^{b}\left(t_{2} n\right)\right] \widetilde{Q}_{v}^{c}(0), \\
J_{3 S}\left(t_{1}, t_{2}\right) & =\epsilon_{a b c}\left[q_{1}^{a \mathrm{~T}}\left(t_{1} n\right) \mathcal{C} q_{2}^{b}\left(t_{2} n\right)\right] \widetilde{Q}_{v}^{c}(0) .
\end{aligned}
$$

Легко заметить, что линейные комбинации токов (15)-(17) и (19)-(21)

$$
\begin{aligned}
2 J_{3 V}^{\mu}\left(t_{1}, t_{2}\right)-\bar{v}^{\mu}\left[J_{2 V}\left(t_{1}, t_{2}\right)-J_{4 V}\left(t_{1}, t_{2}\right)\right] & =2 \epsilon_{a b c}\left[q_{1}^{a \mathrm{~T}}\left(t_{1} n\right) \mathcal{C} \gamma_{t}^{\mu} q_{2}^{b}\left(t_{2} n\right)\right] \widetilde{Q}_{v}^{c}(0), \\
J_{2 T}^{\mu}\left(t_{1}, t_{2}\right)+J_{4 T}^{\mu}\left(t_{1}, t_{2}\right)-2 \bar{v}^{\mu} J_{3 T}\left(t_{1}, t_{2}\right) & =2 \epsilon_{a b c}\left[q_{1}^{a \mathrm{~T}}\left(t_{1} n\right) \mathcal{C} \gamma_{t}^{\mu} \not b q_{2}^{b}\left(t_{2} n\right)\right] \widetilde{Q}_{v}^{c}(0)
\end{aligned}
$$

сводятся к локальным токам с ненулевыми матричными элементами:

$$
\left\langle 0\left|\epsilon_{a b c}\left[q_{1}^{a}(0) \mathcal{C} \gamma_{t}^{\mu} q_{2}^{b}(0)\right] \widetilde{Q}_{v}^{c}(0)\right| \widetilde{H}_{Q}(v, \eta)\right\rangle=\tilde{\lambda}_{1} \eta^{\mu}
$$




$$
\left\langle 0\left|\epsilon_{a b c}\left[q_{1}^{a}(0) \mathcal{C} \gamma_{t}^{\mu} \not b q_{2}^{b}(0)\right] \widetilde{Q}_{v}^{c}(0)\right| \widetilde{H}_{Q}(v, \eta)\right\rangle=\tilde{\lambda}_{2} \eta^{\mu}
$$

Здесь $\bar{v}^{\mu}=\left(n^{\mu}-\bar{n}^{\mu}\right) / 2\left(\bar{v}^{2}=-1\right.$ и $\left.(v \bar{v})=0\right)$ и $\gamma_{t}^{\mu}=\gamma_{\perp}^{\mu}-\not \bar{p} \bar{v}^{\mu}$. Используя введенные выше определения, матричные элементы нелокальных операторов можно определить следующим образом:

$$
\begin{aligned}
\left\langle 0\left|J_{2 V}\left(t_{1}, t_{2}\right)\right| \widetilde{H}_{Q}(v, \eta)\right\rangle & =\tilde{\lambda}_{1}(n \eta) \Psi_{2 V}\left(t_{1}, t_{2}\right), \\
\left\langle 0\left|J_{3 V}^{\mu}\left(t_{1}, t_{2}\right)\right| \widetilde{H}_{Q}(v, \eta)\right\rangle & =\tilde{\lambda}_{1} \eta_{\perp}^{\mu} \Psi_{3 V}\left(t_{1}, t_{2}\right), \\
\left\langle 0\left|J_{4 V}\left(t_{1}, t_{2}\right)\right| \widetilde{H}_{Q}(v, \eta)\right\rangle & =-\tilde{\lambda}_{1}(\bar{n} \eta) \Psi_{4 V}\left(t_{1}, t_{2}\right), \\
\left\langle 0\left|J_{3 A}^{\mu}\left(t_{1}, t_{2}\right)\right| \widetilde{H}_{Q}(v, \eta)\right\rangle & =\tilde{\lambda}_{1} \eta_{\perp}^{\mu} \Psi_{3 A}\left(t_{1}, t_{2}\right), \\
\left\langle 0\left|J_{2 T}^{\mu}\left(t_{1}, t_{2}\right)\right| \widetilde{H}_{Q}(v, \eta)\right\rangle & =\tilde{\lambda}_{2} \eta_{\perp}^{\mu} \Psi_{2 T}\left(t_{1}, t_{2}\right), \\
\left\langle 0\left|J_{3 T}\left(t_{1}, t_{2}\right)\right| \widetilde{H}_{Q}(v, \eta)\right\rangle & =\tilde{\lambda}_{2}(\bar{v} \eta) \Psi_{3 T}\left(t_{1}, t_{2}\right), \\
\left\langle 0\left|J_{4 T}^{\mu}\left(t_{1}, t_{2}\right)\right| \widetilde{H}_{Q}(v, \eta)\right\rangle & =\tilde{\lambda}_{2} \eta_{\perp}^{\mu} \Psi_{4 T}\left(t_{1}, t_{2}\right), \\
\left\langle 0\left|J_{3 S}\left(t_{1}, t_{2}\right)\right| \widetilde{H}_{Q}(v, \eta)\right\rangle & =\tilde{\lambda}_{2}(\bar{v} \eta) \Psi_{3 S}\left(t_{1}, t_{2}\right),
\end{aligned}
$$

где $\eta_{\perp}^{\mu}=\eta^{\mu}+\bar{v}^{\mu}(\bar{v} \eta)$. В пределе $S U(3)_{F}$-симметрии АР $\Psi_{i V}\left(t_{1}, t_{2}\right)$ и $\Psi_{i T}\left(t_{1}, t_{2}\right)$ симметричны относительно замены $t_{1} \leftrightarrow t_{2}$ и имеют нормировку $\Psi_{i V}(0,0)=\Psi_{i T}(0,0)=$ 1 , а $\Psi_{3 S}\left(t_{1}, t_{2}\right)$ и $\Psi_{3 A}\left(t_{1}, t_{2}\right)$ антисимметричны и, следовательно, удовлетворяют условию $\Psi_{3 A}(0,0)=\Psi_{3 S}(0,0)=0$. Нарушение $S U(3)_{F}$-симметрии приводит к нарушению свойств симметрии АР.

Переход к реальному полю тяжелого кварка $Q_{v}^{c}(0)$ в нелокальных токах $(15)-(22)$ осуществляется заменой $\widetilde{Q}_{v}^{c}(0) \rightarrow \Gamma^{\prime} Q_{v}^{c}(0)$, где матрица $\Gamma^{\prime}$ доопределяет требуемые квантовые числа рассматриваемого бариона. Эта замена также меняет и правые части определений (27)-(34), но набор АР остается таким же (детали будут изложены в работе [8]).

\section{3. МАСШТАБНАЯ ЗАВИСИМОСТЬ МАТРИЧНЫХ ЭЛЕМЕНТОВ}

При описании $H_{Q}$-бариона посредством конституэнтных кварков в нерелятивистском приближении предполагается, что $f_{H_{Q}}^{(2)} \simeq f_{H_{Q}}^{(1)}$ при малых энергиях порядка 1 ГэВ, что подтверждается расчетами методом правил сумм KXД [10], [11], [16], [17]. Действительно, разность между двумя параметрами связи возникает только в нелинейно-колебательных (НЛК) пертурбативных поправках к правилам сумм [11], [17], причем численно она мала.

Масштабная зависимость параметров связи задается уравнением

$$
f_{H_{Q}}^{(i)}(\mu)=f_{H_{Q}}^{(i)}\left(\mu_{0}\right)\left(\frac{\alpha_{s}(\mu)}{\alpha_{s}\left(\mu_{0}\right)}\right)^{\gamma_{1}^{(i)} / \beta_{0}}\left[1-\frac{\alpha_{s}\left(\mu_{0}\right)-\alpha_{s}(\mu)}{4 \pi} \frac{\gamma_{1}^{(i)}}{\beta_{0}}\left(\frac{\gamma_{2}^{(i)}}{\gamma_{1}^{(i)}}-\frac{\beta_{1}}{\beta_{0}}\right)\right],
$$

где аномальные размерности локальных интерполяционных операторов

$$
\frac{d \ln f_{H_{Q}}^{(i)}(\mu)}{d \ln \mu} \equiv-\gamma^{(i)}=-\sum_{k} \gamma_{k}^{(i)} a^{k}(\mu), \quad a(\mu) \equiv \frac{\alpha_{s}^{\overline{\mathrm{MS}}}(\mu)}{4 \pi}
$$


известны с НЛК-точностью [17], и в НЛК-разложении $\beta$-функции

$$
\frac{d a(\mu)}{d \ln \mu}=-\beta_{0} a^{2}(\mu)-\beta_{1} a^{3}(\mu)+\cdots
$$

были использованы коэффициенты $\beta_{0}=2\left(11-2 n_{f} / 3\right)$ и $\beta_{1}=4\left(51-19 n_{f} / 3\right)$.

Численно за значения параметров связи примем оценки, полученные из НЛК-анализа правил сумм КХД [7], [11]:

$$
f_{\Lambda_{b}}^{(1)}(1 \Gamma \ni \mathrm{B}) \simeq f_{\Lambda_{b}}^{(2)}(1 \Gamma \ni \mathrm{B}) \simeq(0.030 \pm 0.005) \Gamma \ni \mathrm{B}^{3},
$$

на масштабе перенормировки $\mu=1$ ГэВ. Заметим, что эти параметры различны на произвольном масштабе, поскольку соответствующие им операторы имеют различные аномальные размерности.

AР, введенные в разделе 2, зависят от масштаба. Для определения этой зависимости удобно перейти к импульсному представлению АР, получаемому посредством преобразования Фурье:

$$
\begin{aligned}
\Psi_{k}\left(t_{1}, t_{2}\right) & =\int_{0}^{\infty} d \omega_{1} \int_{0}^{\infty} d \omega_{2} e^{-i\left(t_{1} \omega_{1}+t_{2} \omega_{2}\right)} \psi_{k}\left(\omega_{1}, \omega_{2}\right)= \\
& =\int_{0}^{\infty} \omega d \omega \int_{0}^{1} d u e^{-i \omega\left(t_{1} u+t_{2} \bar{u}\right)} \widetilde{\psi}_{k}(\omega, u)
\end{aligned}
$$

так что $\widetilde{\psi}_{k}(\omega, u)=\psi_{k}(u \omega, \bar{u} \omega)$ и $\bar{u}=1-u$. В первом случае $\omega_{1}$ и $\omega_{2}-$ энергии легких кварков, а во втором введены полная энергия $\omega=\omega_{1}+\omega_{2}$, переносимая легкими кварками (в системе покоя тяжелого кварка), и безразмерная переменная $u$, соответствующая относительной части энергии, переносимой кварком $q_{1}$.

Уравнение эволюции в главном порядке для АР ведущего твиста $\psi_{2}\left(\omega_{1}, \omega_{2} ; \mu\right)$ можно получить, следуя стандартному методу [7] идентификации ультрафиолетовых расходимостей в диаграммах одноглюонного обмена, приведенных на рис. 2. Его удобно записать, используя двухчастичные ядра, возникающие в уравнении эволюции АР для $B$ - и $\pi$-мезонов [7]

$$
\begin{aligned}
\mu \frac{d}{d \mu} \psi_{2}\left(\omega_{1}, \omega_{2} ; \mu\right)= & -\frac{\alpha_{s}(\mu)}{2 \pi}\left(1+\frac{1}{N_{c}}\right)\left\{\int_{0}^{\infty} d \omega_{1}^{\prime} \gamma^{\mathrm{LN}}\left(\omega_{1}^{\prime}, \omega_{1} ; \mu\right) \psi_{2}\left(\omega_{1}^{\prime}, \omega_{2} ; \mu\right)+\right. \\
& +\int_{0}^{\infty} d \omega_{2}^{\prime} \gamma^{\mathrm{LN}}\left(\omega_{2}^{\prime}, \omega_{2} ; \mu\right) \psi_{2}\left(\omega_{1}, \omega_{2}^{\prime} ; \mu\right)- \\
& \left.-\int_{0}^{1} d v V(u, v) \psi_{2}(v \omega, \bar{v} \omega ; \mu)+\frac{3}{2} \psi_{2}\left(\omega_{1}, \omega_{2} ; \mu\right)\right\},
\end{aligned}
$$

где последнее слагаемое $3 \psi_{2}\left(\omega_{1}, \omega_{2} ; \mu\right) / 2$ в фигурных скобках является результатом вычитания однопетлевой перенормировки параметра связи $f_{H_{Q}}^{(2)}$.

Первые две интегральные свертки в формуле (40) описывают динамику в системе из тяжелого и легкого кварков: каждая из них определяется только вкладом одного легкого кварка из пары. Действительно, $\gamma^{\mathrm{LN}}\left(\omega^{\prime}, \omega ; \mu\right)$ совпадает с ядром, определяющим эволюцию АР $B$-мезона, - аномальной размерностью Ланге-Нойберта [18]. В свою очередь, последняя интегральная свертка описывает взаимодействие между легкими кварками, где $V(u, v)$ - хорошо известное ядро Ефремова-Радюшкина-Бродского-Лепажа [19]. 

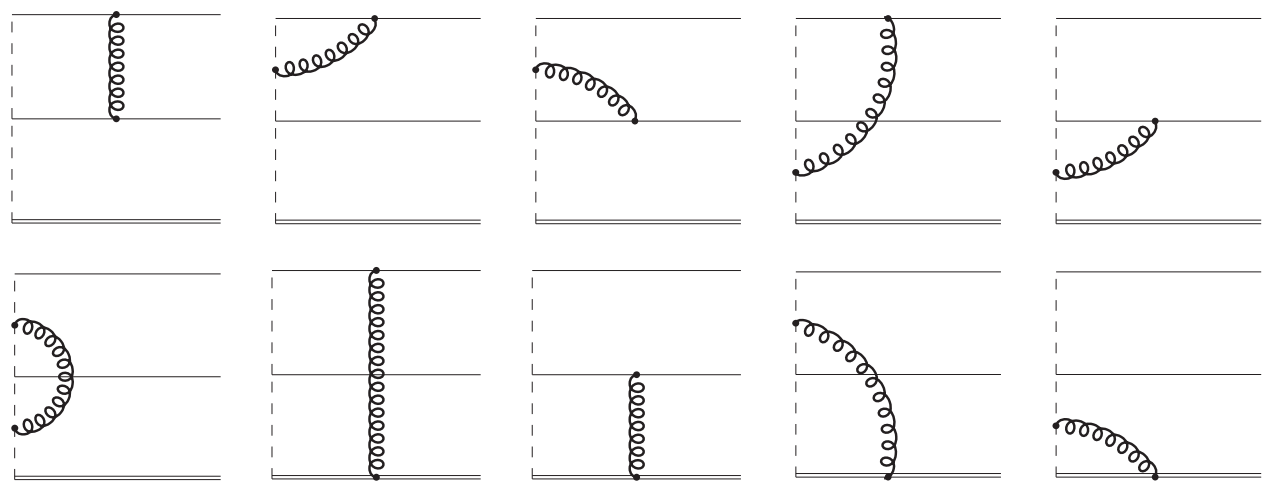

Рис. 2. Полный набор диаграмм одноглюонного обмена, необходимых для вычисления масштабной зависимости АР тяжелого бариона. Одинарные и двойные сплошные линии соответствуют легким и тяжелым кваркам; штриховые и винтовые линии описывают вильсоновские струны и виртуальные глюоны.

Для малых сдвижек по шкале эволюции $\ln \left(\mu / \mu_{0}\right) \lesssim 1$ достаточно заменить производную конечной разностью $\left[\psi_{2}\left(\omega_{1}, \omega_{2} ; \mu\right)-\psi_{2}\left(\omega_{1}, \omega_{2} ; \mu_{0}\right)\right] / \ln \left(\mu / \mu_{0}\right)$ в левой части формулы (40) и подставить граничное условие $\psi_{2}\left(\omega_{1}, \omega_{2} ; \mu_{0}\right)$ вместо $\psi_{2}\left(\omega_{1}, \omega_{2} ; \mu\right)$ в правой части. Очевидно, что это соответствует учету только однопетлевой перенормировки в пренебрежении суммированием потенциально больших логарифмов. Как было показано в работе [7], такое пошаговое (однопетлевое) приближение вполне достаточно для практического применения, т. е. для значений $\mu_{0}=1$ ГэВ и $\mu \simeq m_{b} / 2$, где $m_{b}$ - масса $b$-кварка. Одна из возможностей выхода за рамки однопетлевого приближения состоит в численном интегрировании уравнения эволюции (40). В работе [7] был предложен другой подход (полуаналитический), преимущество которого состоит в том, что можно качественно понять структуру решения.

\section{4. ПРАВИЛА СУММ КХД}

Модельные зависимости АР для тяжелых барионов можно получить, используя правила сумм КХД для корреляционных функций, построенных из нелокального оператора $\mathcal{O}^{\Gamma}\left(t_{1}, t_{2}\right)$ струнного типа и подходящего локального тока $\bar{J}^{\Gamma^{\prime}}(x)$, как показано на рис. 3 . В качестве локального тока тяжелого бариона можно выбрать

$$
\bar{J}^{\Gamma^{\prime}}(x)=A \bar{J}_{1}^{H_{Q}}+B \bar{J}_{2}^{H_{Q}}=\varepsilon_{a b c}\left[\bar{q}_{2}^{a}(x)(A+B \not b) \Gamma^{\prime} \mathcal{C}^{\mathrm{T}} \bar{q}_{1}^{b \mathrm{~T}}(x)\right] \bar{Q}_{v}^{c}(x),
$$

где $\mathcal{C}^{\mathrm{T}}$ - транспонированная матрица $\mathcal{C}$. Варьируя значения коэффициентов $A$ и $B$, можно оценить неопределенность, обусловленную выбором локального тока. Токи $\bar{J}_{1}^{H_{Q}}(x)$ и $\bar{J}_{2}^{H_{Q}}(x)$ были использованы в работе [11] для построения диагональных, недиагональных и конституэнтного типа правил сумм. Для бариона со спин-четностью дикварка $j^{p}=0^{+}$нужно взять $\Gamma^{\prime}=\gamma_{5}$, а для $j^{p}=1^{+}$дикварка имеются две возможности: $\Gamma^{\prime}=\gamma_{\|}$или $\Gamma^{\prime}=\gamma_{\perp}$. Ток $\bar{J}_{\Lambda}(x)$, выбранный в работе [7] для $\Lambda_{b}$-бариона, имеющего $j^{p}=0^{+}$у дикварка, есть не что иное, как ток (41) 


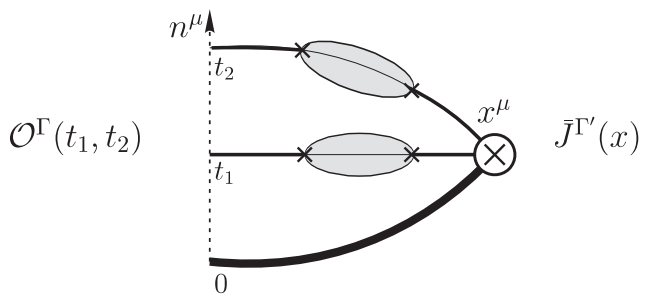

Рис. 3. Корреляционные функции, построенные из нелокального оператора $\mathcal{O}^{\Gamma}\left(t_{1}, t_{2}\right)$ струнного типа и локального тока $\bar{J}^{\Gamma^{\prime}}(x)$. Жирная сплошная линия соответствует пропагатору тяжелого кварка, а сплошные линии со вставками в виде эллипсов - пропагаторам легких кварков, модифицированным вкладами нелокальных кварковых конденсатов.

с $A=B=1 / 2$, который учитывает вклады как четной, так и нечетной размерностей, т. е. $\bar{J}_{2}^{\Lambda_{b}}(x)$ индуцирует вклады, обусловленные пертурбативной теорией и четырехфермионным конденсатом, в то время как $\bar{J}_{1}^{\Lambda_{b}}(x)$ приводит к появлению нелокальных кварковых конденсатов.

Правила построения правил сумм КХД хорошо известны и приводят к следующему общему выражению:

$$
f_{k}\left(A f_{H_{Q}}^{(1)}+B f_{H_{Q}}^{(2)}\right) \widetilde{\psi}_{k}^{\mathrm{SR}}\left(t_{1}, t_{2}\right) e^{-\bar{\Lambda} / \tau}=\mathcal{B}\left[\Pi_{k}\right]\left(t_{1}, t_{2} ; \tau, s_{0}\right),
$$

где $f_{k}=f_{H_{Q}}^{(2)}$ для АР четных твистов и $f_{k}=f_{H_{Q}}^{(1)}$ для АР твиста-3. Эффективная масса бариона вводится как разность $\bar{\Lambda}=m_{H_{Q}}-m_{Q}$, где $m_{Q}$ - масса тяжелого кварка, $\tau$ - борелевский параметр и $s_{0}$ - порог континуума. Правая часть уравнения (42) - преобразованная по Борелю инвариантная функция без вклада континуума, определяемая посредством коррелятора $\Pi_{k}\left(t_{1}, t_{2} ; \tau\right)$.

Для практического использования необходимо знать АР в пространстве импульсов. Преобразование Фурье АР было определено формулой (39), а для скалярной корреляционной функции примем следующее определение:

$$
\Pi_{k}(\omega, u ; \tau)=\int_{-\infty}^{\infty} \frac{d t_{1}}{2 \pi} \int_{-\infty}^{\infty} \frac{d t_{2}}{2 \pi} e^{i \omega\left(u t_{1}+\bar{u} t_{2}\right)} \Pi_{k}\left(t_{1}, t_{2} ; \tau\right) .
$$

В импульсном пространстве правила сумм (42) могут быть представлены в виде

$$
f_{k}\left(A f_{H_{Q}}^{(1)}+B f_{H_{Q}}^{(2)}\right) \widetilde{\psi}_{k}^{\mathrm{SR}}(\omega, u) e^{-\bar{\Lambda} / \tau}=\mathcal{B}\left[\Pi_{k}\right]\left(\omega, u ; \tau, s_{0}\right) .
$$

Учитывая только пертурбативный вклад низшего порядка в правилах сумм, получим

$$
\begin{aligned}
& \widetilde{\psi}_{2}(\omega, u)=\frac{30 \tau^{4}}{\mathcal{N}}\left[\widehat{\omega}^{2} u \bar{u}+\frac{A}{B} \widehat{\omega}\left(\widehat{m}_{2} u+\widehat{m}_{1} \bar{u}\right)\right] E_{1}\left(2 \hat{s}_{\omega}\right) e^{-\widehat{\omega}}, \\
& \widetilde{\psi}_{4}(\omega, u)=\frac{30 \tau^{4}}{\mathcal{N}}\left[E_{3}\left(2 \hat{s}_{\omega}\right)+\frac{A}{B}\left(\widehat{m}_{1}+\widehat{m}_{2}\right) E_{2}\left(2 \hat{s}_{\omega}\right)\right] e^{-\widehat{\omega}},
\end{aligned}
$$




$$
\begin{aligned}
\widetilde{\psi}_{3 s}(\omega, u)= & \frac{15 \tau^{4}}{\mathcal{N}}\left\{\left[\widehat{\omega}+\frac{B}{A}\left(\widehat{m}_{1}+\widehat{m}_{2}\right)\right] E_{2}\left(2 \hat{s}_{\omega}\right)+\right. \\
& \left.+\frac{B}{A} \widehat{\omega}\left(\widehat{m}_{2} u+\widehat{m}_{1} \bar{u}\right) E_{1}\left(2 \hat{s}_{\omega}\right)\right\} e^{-\widehat{\omega}}, \\
\widetilde{\psi}_{3 \sigma}(\omega, u) & =\frac{15 \tau^{4}}{\mathcal{N}}\left\{\left[\widehat{\omega}(u-\bar{u})+\frac{B}{A}\left(\widehat{m}_{1}-\widehat{m}_{2}\right)\right] E_{2}\left(2 \hat{s}_{\omega}\right)+\right. \\
& \left.+\frac{B}{A} \widehat{\omega}\left(\widehat{m}_{2} u-\widehat{m}_{1} \bar{u}\right) E_{1}\left(2 \hat{s}_{\omega}\right)\right\} e^{-\widehat{\omega}},
\end{aligned}
$$

где $s_{\omega}=s_{0}-\omega / 2, \widehat{\omega}=\omega /(2 \tau), \hat{s}_{\omega}=s_{\omega} /(2 \tau)$ и $\widehat{m}_{1,2}=m_{1,2} /(2 \tau)$. Нормировочный интеграл $\mathcal{N}$ можно выразить через параметр связи бариона:

$$
\left|f_{H_{Q}}^{(i)}\right|^{2} e^{-\bar{\Lambda} / \tau}=\frac{1}{20 \pi^{4}} \int_{0}^{s_{0}} d s s^{5} e^{-s / \tau} \equiv \frac{\mathcal{N}}{20 \pi^{4}} .
$$

Для удобства записи уравнений (45)-(48) была введена функция

$$
E_{a}(x)=\frac{1}{\Gamma(a+1)} \int_{0}^{x} d t t^{a} e^{-t}=1-\frac{\Gamma(a+1, x)}{\Gamma(a+1)},
$$

которая выражается через неполную Г-функцию $\Gamma(a+1, x)=\int_{x}^{\infty} d t t^{a} e^{-t}$. Для целых значений параметра $(a=N)$ эта функция сводится к хорошо известному виду

$$
E_{N}(x)=1-e^{-x} \sum_{n=0}^{N} \frac{x^{n}}{n !} .
$$

При проведении численных расчетов удобно воспользоваться интегрированием по частям:

$$
E_{a}(x)=E_{a+1}(x)+\frac{x^{a+1} e^{-x}}{\Gamma(a+2)},
$$

чтобы преобразовать отрицательное значение $a$ в функции $E_{a}(x)$ в положительное, как это было сделано в работе [7].

Чтобы оценить непертурбативные вклады в правила сумм, можно воспользоваться техникой нелокальных кварковых конденсатов, примененной к $B$-мезону в работах [20], [21]. При проведении вычислений было использовано общее определение нелокального кваркового конденсата [22], [23]

$$
\langle\bar{q}(x) q(0)\rangle=\langle\bar{q} q\rangle \int_{0}^{\infty} d \nu e^{\nu x^{2} / 4} f(\nu)
$$

где $\langle\bar{q} q\rangle$ - локальный кварковый конденсат и $f(\nu)$ - модельная функция [21], [24]

$$
f(\nu)=\frac{\lambda^{a-2}}{\Gamma(a-2)} \nu^{1-a} e^{-\lambda / \nu}, \quad a-3=\frac{4 \lambda}{m_{0}^{2}} .
$$

Параметры $\lambda$ и $m_{0}^{2}$, входящие в модельную функцию $f(\nu)$, имеют смысл длины корреляции и отношения локальных смешанного кварк-глюонного и кваркового конденсатов. Исчерпывающий анализ нелокальных кварковых и глюонных конденсатов высших размерностей представлен в работе [25]. 
Используя нелокальные кварковые конденсаты, получим следующие правила сумм:

$$
\begin{aligned}
& f_{H_{Q}}^{(2)}\left(A f_{H_{Q}}^{(1)}+B f_{H_{Q}}^{(2)}\right) \widetilde{\psi}_{2}^{\mathrm{SR}}(\omega, u) e^{-\bar{\Lambda} / \tau}= \\
& =\frac{3 \tau^{4}}{2 \pi^{4}}\left[B \widehat{\omega}^{2} u \bar{u}\left(\widehat{\omega}^{2} u \bar{u}+\widehat{m}_{1} \widehat{m}_{2}\right)+A \widehat{\omega}\left(\widehat{m}_{2} u+\widehat{m}_{1} \bar{u}\right)\right] E_{1}\left(2 \hat{s}_{\omega}\right) e^{-\widehat{\omega}}- \\
& -\frac{\left\langle\bar{q}_{1} q_{1}\right\rangle \tau^{3}}{\pi^{2}}\left[A \widehat{\omega} \bar{u}+B \widehat{m}_{2}\right] f(2 \tau \omega u) E_{2-a}\left(2 \hat{s}_{\kappa}\right) e^{-\widehat{\omega}}- \\
& -\frac{\left\langle\bar{q}_{2} q_{2}\right\rangle \tau^{3}}{\pi^{2}}\left[A \widehat{\omega} u+B \widehat{m}_{1}\right] f(2 \tau \omega \bar{u}) E_{2-a}\left(2 \hat{s}_{\bar{\kappa}}\right) e^{-\widehat{\omega}}+ \\
& +\frac{2 B}{3}\left\langle\bar{q}_{1} q_{1}\right\rangle\left\langle\bar{q}_{2} q_{2}\right\rangle \tau^{2} f(2 \tau \omega u) f(2 \tau \omega \bar{u}) E_{3-2 a}\left(2 \hat{s}_{\kappa \bar{\kappa}}\right) e^{-\widehat{\omega}}, \\
& f_{H_{Q}}^{(2)}\left(A f_{H_{Q}}^{(1)}+B f_{H_{Q}}^{(2)}\right) \widetilde{\psi}_{4}^{\mathrm{SR}}(\omega, u) e^{-\bar{\Lambda} / \tau}= \\
& =\frac{3 \tau^{4}}{2 \pi^{4}}\left[B E_{3}\left(2 \hat{s}_{\omega}\right)+A\left(\widehat{m}_{1}+\widehat{m}_{2}\right) E_{2}\left(2 \hat{s}_{\omega}\right)\right] e^{-\widehat{\omega}}- \\
& -\frac{\left\langle\bar{q}_{1} q_{1}\right\rangle \tau^{3}}{\pi^{2}}\left[A E_{3-a}\left(2 \hat{s}_{\kappa}\right)+B \widehat{m}_{2} E_{2-a}\left(2 \hat{s}_{\kappa}\right)\right] f(2 \tau \omega u) e^{-\widehat{\omega}}- \\
& -\frac{\left\langle\bar{q}_{2} q_{2}\right\rangle \tau^{3}}{\pi^{2}}\left[A E_{3-a}\left(2 \hat{s}_{\bar{\kappa}}\right)+B \widehat{m}_{1} E_{2-a}\left(2 \hat{s}_{\bar{\kappa}}\right)\right] f(2 \tau \omega \bar{u}) e^{-\widehat{\omega}}+ \\
& +\frac{2 B}{3}\left\langle\bar{q}_{1} q_{1}\right\rangle\left\langle\bar{q}_{2} q_{2}\right\rangle \tau^{2} f(2 \tau \omega u) f(2 \tau \omega \bar{u}) E_{3-2 a}\left(2 \hat{s}_{\kappa \bar{\kappa}}\right) e^{-\widehat{\omega}}, \\
& f_{H_{Q}}^{(1)}\left(A f_{H_{Q}}^{(1)}+B f_{H_{Q}}^{(2)}\right) \widetilde{\psi}_{3 s}^{\mathrm{SR}}(\omega, u) e^{-\bar{\Lambda} / \tau}= \\
& =\frac{3 \tau^{4}}{4 \pi^{4}}\left\{\left[A \widehat{\omega}+B\left(\widehat{m}_{1}+\widehat{m}_{2}\right)\right] E_{2}\left(2 \hat{s}_{\omega}\right)+B \widehat{\omega}\left(\widehat{m}_{2} u+\widehat{m}_{1} \bar{u}\right) E_{1}\left(2 \hat{s}_{\omega}\right)\right\} e^{-\widehat{\omega}}- \\
& -\frac{\left\langle\bar{q}_{1} q_{1}\right\rangle \tau^{3}}{2 \pi^{2}}\left[B E_{3-a}\left(2 \hat{s}_{\kappa}\right)+\left(B \widehat{\omega} \bar{u}+2 A \widehat{m}_{2}\right) E_{2-a}\left(2 \hat{s}_{\kappa}\right)\right] f(2 \tau \omega u) e^{-\widehat{\omega}}- \\
& -\frac{\left\langle\bar{q}_{2} q_{2}\right\rangle \tau^{3}}{2 \pi^{2}}\left[B E_{3-a}\left(2 \hat{s}_{\bar{\kappa}}\right)+\left(B \widehat{\omega} u+2 A \widehat{m}_{1}\right) E_{2-a}\left(2 \hat{s}_{\bar{\kappa}}\right)\right] f(2 \tau \omega \bar{u}) e^{-\widehat{\omega}}+ \\
& +\frac{2 A}{3}\left\langle\bar{q}_{1} q_{1}\right\rangle\left\langle\bar{q}_{2} q_{2}\right\rangle \tau^{2} f(2 \tau \omega u) f(2 \tau \omega \bar{u}) E_{3-2 a}\left(2 \hat{s}_{\kappa \bar{\kappa}}\right) e^{-\widehat{\omega}}, \\
& f_{H_{Q}}^{(1)}\left(A f_{H_{Q}}^{(1)}+B f_{H_{Q}}^{(2)}\right) \widetilde{\psi}_{3 \sigma}^{\mathrm{SR}}(\omega, u) e^{-\bar{\Lambda} / \tau}= \\
& =\frac{3 \tau^{4}}{4 \pi^{4}}\left\{\left[A \widehat{\omega}(u-\bar{u})+B\left(\widehat{m}_{1}-\widehat{m}_{2}\right)\right] E_{2}\left(2 \hat{s}_{\omega}\right)+B \widehat{\omega}\left(\widehat{m}_{2} u-\widehat{m}_{1} \bar{u}\right) E_{1}\left(2 \hat{s}_{\omega}\right)\right\} e^{-\widehat{\omega}}- \\
& -\frac{B\left\langle\bar{q}_{1} q_{1}\right\rangle \tau^{3}}{2 \pi^{2}}\left[E_{3-a}\left(2 \hat{s}_{\kappa}\right)-\widehat{\omega} \bar{u} E_{2-a}\left(2 \hat{s}_{\kappa}\right)\right] f(2 \tau \omega u) e^{-\widehat{\omega}}+ \\
& +\frac{B\left\langle\bar{q}_{2} q_{2}\right\rangle \tau^{3}}{2 \pi^{2}}\left[E_{3-a}\left(2 \hat{s}_{\bar{\kappa}}\right)-\widehat{\omega} u E_{2-a}\left(2 \hat{s}_{\bar{\kappa}}\right)\right] f(2 \tau \omega \bar{u}) e^{-\widehat{\omega}},
\end{aligned}
$$

где $\hat{s}_{\kappa}=\hat{s}_{\omega}-\kappa / 2, \hat{s}_{\bar{\kappa}}=\hat{s}_{\omega}-\bar{\kappa} / 2, \hat{s}_{\kappa \bar{\kappa}}=\hat{s}_{\omega}-\kappa / 2-\bar{\kappa} / 2, \kappa=\lambda /(2 u \omega \tau), \bar{\kappa}=\lambda /(2 \bar{u} \omega \tau)$. КХД-правила сумм для АР твиста-2 (55) совпадают с полученными в работе [7] в пределе безмассовых легких кварков $\widehat{m}_{1}=\widehat{m}_{2}=0$, когда $A=B=1 / 2$. Эффект кварк-адронной дуальности в квадратичном по кварковому конденсату слагаемом в формулах (55)-(57) приводит к появлению функции $E_{3-2 a}\left(2 \hat{s}_{\kappa \bar{\kappa}}\right)$, которая в локальном пределе обращается в единицу в согласии с оригинальным результатом [7]. 
Аналогичным образом можно построить КХД-правила сумм для тяжелых барионов, содержащих дикварк со спин-четностью $j^{p}=1^{+}$. Соответствующие формулы будут приведены в работе [8].

\section{5. ЧИСЛЕННЫЙ АНАЛИЗ}

Для проведения численного анализа необходимо определиться со значениями входных параметров. Эффективные массы барионов $\bar{\Lambda}=m_{H_{b}}-m_{b}$ в ЭТТК для $m_{b}=4.8$ ГэВ приведены в табл. 1, где представлены экспериментальные данные [26] и теоретические предсказания, полученные в ЭТТК [27] и решеточной КХД [28], для масс (в единицах МэВ) $b$-барионов основного состояния. Сравнительный анализ предсказаний для масс тяжелых барионов может быть найден в работах [27], [29]. Приведенные значения порога континуума $s_{0}$ (последняя колонка в табл. 1) согласуются с оценками, использованными для вычисления масс барионов, с точностью $1 / m_{b}$ в рамках ЭТТК. Значения параметров, входящих в правила сумм КХД для AP $b$-барионов, приведены в табл. 2. Обсуждение этих параметров можно найти в работе [30] и приведенных там ссылках. Отметим, что модельная функция $f(\nu)$ в нелокальном кварковом конденсате (53) предполагается не зависящей от аромата легких кварков.

ТАБЛИцА 1

\begin{tabular}{ccccccc}
\hline Барион & $J^{P}$ & Эксперимент [26] & ЭТТК [27] & Решеточная КХД $[28]$ & $\bar{\Lambda}$ & $s_{0}$ \\
\hline$\Lambda_{b}$ & $1 / 2^{+}$ & $5620.0 \pm 1.6$ & $5637_{-56}^{+68}$ & $5641 \pm 21_{-33}^{+15}$ & 0.8 & 1.2 \\
$\Sigma_{b}^{+}$ & $1 / 2^{+}$ & $5807.8 \pm 2.7$ & $5809_{-76}^{+82}$ & $5795 \pm 16_{-26}^{+17}$ & 1.0 & 1.3 \\
$\Sigma_{b}^{-}$ & $1 / 2^{+}$ & $5815.2 \pm 2.0$ & $5809_{-76}^{+82}$ & $5795 \pm 16_{-26}^{+17}$ & 1.0 & 1.3 \\
$\Sigma_{b}^{*+}$ & $3 / 2^{+}$ & $5829.0 \pm 3.4$ & $5835_{-77}^{+82}$ & $5842 \pm 26_{-18}^{+20}$ & 1.0 & 1.3 \\
$\Sigma_{b}^{*-}$ & $3 / 2^{+}$ & $5836.4 \pm 2.8$ & $5835_{-77}^{+82}$ & $5842 \pm 26_{-18}^{+20}$ & 1.0 & 1.3 \\
$\Xi_{b}^{-}$ & $1 / 2^{+}$ & $5790.5 \pm 2.7$ & $5780_{-68}^{+73}$ & $5781 \pm 17_{-16}^{+17}$ & 1.0 & 1.3 \\
$\Xi_{b}^{\prime}$ & $1 / 2^{+}$ & & $5903_{-79}^{+81}$ & $5903 \pm 12_{-19}^{+18}$ & 1.1 & 1.4 \\
$\Xi_{b}^{\prime *}$ & $3 / 2^{+}$ & & $5903_{-79}^{+81}$ & $5950 \pm 21_{-21}^{+19}$ & 1.1 & 1.4 \\
$\Omega_{b}^{-}$ & $1 / 2^{+}$ & $6071 \pm 40$ & $6036 \pm 81$ & $6006 \pm 10_{-19}^{+20}$ & 1.3 & 1.5 \\
$\Omega_{b}^{*}$ & $3 / 2^{+}$ & & $6063_{-82}^{+83}$ & $6044 \pm 18_{-21}^{+20}$ & 1.3 & 1.5 \\
\hline
\end{tabular}

ТАБЛИЦА 2

\begin{tabular}{lc|lc}
\hline$\tau$ & $(0.6 \pm 0.2) \Gamma \ni \mathrm{B}$ & $m_{s}(1 \Gamma \ni \mathrm{B})$ & $(128 \pm 21) \mathrm{M \ni B}$ \\
$\langle\bar{q} q\rangle(1 \Gamma \ni \mathrm{B})$ & $-\left(242_{-19}^{+28}\right) \mathrm{M \ni B}^{3}$ & $\langle\bar{s} s\rangle /\langle\bar{q} q\rangle$ & $0.8 \pm 0.2$ \\
$m_{0}^{2}$ & $(0.8 \pm 0.2) \Gamma_{\ni} \mathrm{B}^{2}$ & $\lambda$ & $0.16 \Gamma_{\ni} \mathrm{B}^{2}$ \\
\hline
\end{tabular}


С помощью этих правил сумм можно вычислить моменты АР - определенные интегралы от доли импульса, уносимой кварками. Определим моменты АР следующим образом:

$$
\langle f(\omega, u)\rangle_{t}^{H_{Q}} \equiv \int_{0}^{2 s_{0}} \omega d \omega \int_{0}^{1} d u f(\omega, u) \widetilde{\psi}_{t}^{\mathrm{SR}}(\omega, u),
$$

где $t=2,3 s, 3 \sigma, 4$.

Нормировка АР ведущего твиста $(t=2)$ фиксируется условием

$$
\int_{0}^{2 s_{0}} \omega d \omega \int_{0}^{1} d u \widetilde{\psi}_{2}^{\mathrm{SR}}(\omega, u) \equiv 1 .
$$

Для АР твиста-2 удобно делать разложение по полиномам Гегенбауэра $C_{n}^{3 / 2}(2 u-1)$, ортогональным с весом, совпадающим с асимптотикой АР ведущего твиста $\sim u(1-u)$ (55) (в безмассовом пределе). Ошибки соответствуют изменению параметров $A$ и $B$ на отрезке: $0 \leqslant A, B \leqslant 1$, учитывая, что $A+B=1$, а центральные значения остальных входных параметров взяты из табл. 2. Для проверки правильности расчетов нами были воспроизведены численные значения моментов [7] для АР ведущего твиста $\Lambda_{b}$-бариона. Все моменты $\Lambda_{b}$-бариона, вычисленные по отношению к полиномам Гегенбауэра нечетного порядка, обращаются в нуль вследствие симметрии функции при замене $u \leftrightarrow 1-u$. Это не будет выполняться для $\Xi_{b}$-бариона, если учесть поправки, нарушающие $S U(3)_{F}$-симметрию и обусловленные ненулевой массой $s$-кварка и разностью в значениях странного $\langle\bar{s} s\rangle$ и нестранного $\langle\bar{q} q\rangle$ локальных конденсатов. Легко заметить (см. табл. 3), что типичная величина этих поправок составляет $\sim 10 \%$.

Для $\mathrm{AP}$ твиста-3 $\widetilde{\psi}_{3 s}^{\mathrm{SR}}(\omega, u)$ и твиста-4 $\widetilde{\psi}_{4}^{\mathrm{SR}}(\omega, u)$ также использовано условие нормировки (60). Интеграл $(60)$ с $\mathrm{AP} \widetilde{\psi}_{3 \sigma}^{\mathrm{SR}}(\omega, u)$ обращается в нуль в пределе $S U(3)_{F^{-}}$ симметрии, так как эта функция антисимметрична при замене $u \leftrightarrow 1-u$. По этой причине для $\mathrm{AP} \widetilde{\psi}_{3 \sigma}^{\mathrm{SR}}(\omega, u)$ была использована следующая нормировка:

$$
\int_{0}^{2 s_{0}} \omega d \omega \int_{0}^{1} d u C_{1}^{1 / 2}(2 u-1) \widetilde{\psi}_{3 \sigma}^{\mathrm{SR}}(\omega, u) \equiv 1 .
$$

Отметим, что для этих АР более пригодно разложение по полиномам Гегенбауэра $C_{n}^{1 / 2}(2 u-1)$. Это обусловлено анализом случая $\pi$-мезона большой энергии, для которого АР твиста-3 $\phi_{p}(u)$ и твиста- $4 g_{\pi}(u)$ описываются полиномами Гегенбауэра $C_{n}^{1 / 2}(2 u-1)[31]-[33]$.

Численные оценки моментов для $\Lambda_{b^{-}}$и $\Xi_{b^{-}}$-барионов на основе правил сумм КХД представлены в табл. 3. Моменты $\left\langle\omega^{-1}\right\rangle,\left\langle\omega^{-1} C_{n}^{3 / 2}\right\rangle$ и $\left\langle\omega^{-1} C_{n}^{1 / 2}\right\rangle$ имеют размерность Гэ $\mathrm{B}^{-1}$, а $\left\langle C_{n}^{3 / 2}\right\rangle$ и $\left\langle C_{n}^{1 / 2}\right\rangle$ безразмерны. Как объяснялось выше, ошибки соответствуют изменению параметра $A(B=1-A)$ при условии, что все остальные параметры фиксированы и принимают свои центральные значения из табл. 2. Следует отметить, что для $\Xi_{b}$-бариона интеграл $(60)$ с $\widetilde{\psi}_{3 \sigma}^{\mathrm{SR}}(\omega, u)$ не равен нулю вследствие нарушения $S U(3)_{F}$-симметрии, хотя и мал численно:

$$
\int_{0}^{2 s_{0}} \omega d \omega \int_{0}^{1} d u \widetilde{\psi}_{3 \sigma}^{\mathrm{SR}}(\omega, u)=-0.0049_{-0.0037}^{+0.0049} .
$$


ТАБЛИЦА 3

\begin{tabular}{c|c|ccccccc}
\hline \hline$H_{Q}$ & $t$ & $\left\langle\omega^{-1}\right\rangle$ & $\left\langle C_{1}^{3 / 2}\right\rangle$ & $\left\langle\omega^{-1} C_{1}^{3 / 2}\right\rangle$ & $\left\langle C_{2}^{3 / 2}\right\rangle$ & $\left\langle\omega^{-1} C_{2}^{3 / 2}\right\rangle$ & $\left\langle C_{3}^{3 / 2}\right\rangle$ & $\left\langle\omega^{-1} C_{3}^{3 / 2}\right\rangle$ \\
\hline$\Lambda_{b}$ & 2 & $1.65_{-0.47}^{+0.91}$ & 0 & 0 & $1.00_{-1.03}^{+0.54}$ & $0.61_{-1.45}^{+0.76}$ & 0 & 0 \\
$\Xi_{b}$ & 2 & $1.46_{-0.34}^{+0.54}$ & $0.10_{-0.06}^{+0.10}$ & $0.08_{-0.05}^{+0.07}$ & $1.15_{-0.98}^{+0.61}$ & $0.86_{-1.10}^{+0.68}$ & $-0.02_{-0.52}^{+0.32}$ & $-0.10_{-0.38}^{+0.24}$ \\
\hline \hline$H_{Q}$ & $t$ & $\left\langle\omega^{-1}\right\rangle$ & $\left\langle C_{1}^{1 / 2}\right\rangle$ & $\left\langle\omega^{-1} C_{1}^{1 / 2}\right\rangle$ & $\left\langle C_{2}^{1 / 2}\right\rangle$ & $\left\langle\omega^{-1} C_{2}^{1 / 2}\right\rangle$ & $\left\langle C_{3}^{1 / 2}\right\rangle$ & $\left\langle\omega^{-1} C_{3}^{1 / 2}\right\rangle$ \\
\hline & $3 \mathrm{~s}$ & $2.16_{-0.36}^{+0.70}$ & 0 & 0 & $-0.032_{-0.041}^{+0.022}$ & $-0.29_{-0.27}^{+0.14}$ & 0 & 0 \\
$\Lambda_{b}$ & $3 \sigma$ & 0 & 1 & $1.54_{-0.22}^{+0.14}$ & 0 & 0 & $-0.034_{-0.021}^{+0.034}$ & $-0.027_{-0.017}^{+0.027}$ \\
& 4 & $2.84_{-0.46}^{+0.88}$ & 0 & 0 & $-0.108_{-0.018}^{+0.035}$ & $-0.41_{-0.15}^{+0.08}$ & 0 & 0 \\
\hline & $3 \mathrm{~s}$ & $1.94_{-0.21}^{+0.33}$ & $0.11_{-0.05}^{+0.09}$ & $0.075_{-0.047}^{+0.077}$ & $1.05_{-0.23}^{+0.14}$ & $1.01_{-0.46}^{+0.28}$ & $-0.014_{-0.032}^{+0.051}$ & $-0.117_{-0.005}^{+0.002}$ \\
$\Xi_{b}$ & $3 \sigma$ & $0.0019_{-0.0019}^{+0.0014}$ & 1 & $1.37_{-0.14}^{+0.11}$ & $0.057_{-0.057}^{+0.043}$ & $0.098_{-0.098}^{+0.075}$ & $1.11_{-0.35}^{+0.46}$ & $1.55_{-0.32}^{+0.24}$ \\
& 4 & $2.73_{-0.35}^{+0.61}$ & $0.12_{-0.05}^{+0.09}$ & $0.05_{-0.05}^{+0.09}$ & $0.55_{-0.11}^{+0.18}$ & $0.99_{-0.09}^{+0.16}$ & $-0.043_{-0.015}^{+0.025}$ & $-0.18_{-0.03}^{+0.02}$ \\
\hline
\end{tabular}

То же самое справедливо и для момента $\left\langle\omega^{-1}\right\rangle$, как это следует из табл. 3.

Нами предложены следующие простые модели для АР барионов на низком масштабе $\mu=1$ ГэВ:

$$
\begin{aligned}
\widetilde{\psi}_{2}(\omega, u) & =\omega^{2} u(1-u) \sum_{n=0}^{2} \frac{a_{n}^{(2)}}{\epsilon_{n}^{(2)}} C_{n}^{3 / 2}(2 u-1) e^{-\omega / \epsilon_{n}^{(2)}} \\
\widetilde{\psi}_{3 s}(\omega, u) & =\frac{\omega}{2} \sum_{n=0}^{2} \frac{a_{n}^{(3)}}{\epsilon_{n}^{(3)^{3}}} C_{n}^{1 / 2}(2 u-1) e^{-\omega / \epsilon_{n}^{(3)}} \\
\widetilde{\psi}_{3 \sigma}(\omega, u) & =\frac{\omega}{2} \sum_{n=0}^{3} \frac{b_{n}^{(3)}}{\eta_{n}^{(3)^{3}}} C_{n}^{1 / 2}(2 u-1) e^{-\omega / \eta_{n}^{(3)}} \\
\widetilde{\psi}_{4}(\omega, u) & =\sum_{n=0}^{2} \frac{a_{n}^{(4)}}{\epsilon_{n}^{(4)}} C_{n}^{1 / 2}(2 u-1) e^{-\omega / \epsilon_{n}^{(4)}}
\end{aligned}
$$

где $C_{0}^{1 / 2}(2 u-1)=C_{0}^{3 / 2}(2 u-1)=1$. Гегенбауэровские моменты нулевого порядка определяются как $a_{0}^{(t)} \equiv 1$, что следует из нормировки

$$
\int_{0}^{\infty} \omega d \omega \int_{0}^{1} d u \widetilde{\psi}_{t}(\omega, u) \equiv 1
$$

где $t=2,3 s, 4$. При построении моделей АР нами удержаны только два неасимптотических члена в гегенбауэровском разложении. Значения моментов, вычисленных на основе правил сумм КХД, были использованы для оценки величин параметров, входящих в теоретические модели (63)-(66) (см. табл. 4). 

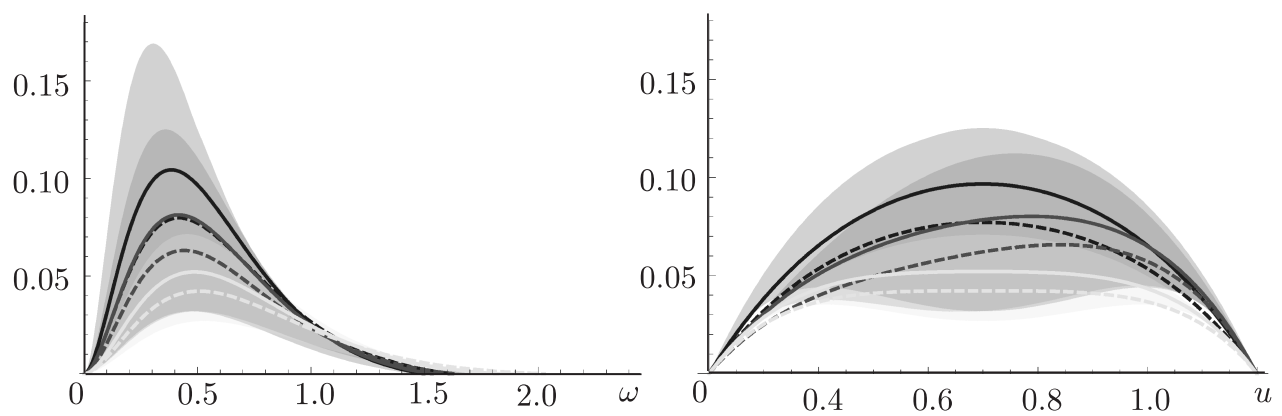

Рис. 4. $\mathrm{AP}$ твиста-2 для $\Sigma_{b}, \Xi_{b}$ и $\Omega_{b}$, построенные для энергий $\mu=1$ ГэВ (сплошные линии) и $\mu=2.5$ ГэВ (штриховые линии), с учетом наиболее консервативной оценки $A \in[0,1]$ (светлые затемненные области).

Интересующие нас моменты, выраженные через параметры моделей (63)-(66), равны

$$
\begin{aligned}
\left\langle\omega^{-1}\right\rangle_{t}^{H_{Q}} & =\left\{\frac{1}{3 \epsilon_{0}^{(2)}}, \frac{1}{2 \epsilon_{0}^{(3)}}, \frac{b_{0}^{(3)}}{2 \eta_{0}^{(3)}}, \frac{1}{\epsilon_{0}^{(4)}}\right\}, \\
\left\langle C_{1}^{3 / 2}\right\rangle_{t}^{H_{Q}} & =\left\{\frac{9 a_{1}^{(2)}}{5}, \frac{a_{1}^{(3)}}{3}, \frac{b_{1}^{(3)}}{3}, \frac{a_{1}^{(4)}}{3}\right\}, \\
\left\langle\omega^{-1} C_{1}^{3 / 2}\right\rangle_{t}^{H_{Q}} & =\left\{\frac{3 a_{1}^{(2)}}{5 \epsilon_{1}^{(2)}}, \frac{a_{1}^{(3)}}{6 \epsilon_{1}^{(3)}}, \frac{b_{1}^{(3)}}{6 \eta_{1}^{(3)}}, \frac{a_{1}^{(4)}}{3 \epsilon_{1}^{(4)}}\right\}, \\
\left\langle C_{2}^{3 / 2}\right\rangle_{t}^{H_{Q}} & =\left\{\frac{18 a_{2}^{(2)}}{7}, \frac{a_{2}^{(3)}}{5}, \frac{b_{2}^{(3)}}{5}, \frac{a_{2}^{(4)}}{5}\right\}, \\
\left\langle\omega^{-1} C_{2}^{3 / 2}\right\rangle_{t}^{H_{Q}} & =\left\{\frac{6 a_{2}^{(2)}}{7 \epsilon_{2}^{(2)}}, \frac{a_{2}^{(3)}}{10 \epsilon_{2}^{(3)}}, \frac{b_{2}^{(3)}}{10 \eta_{2}^{(3)}}, \frac{a_{2}^{(4)}}{5 \epsilon_{2}^{(4)}}\right\} .
\end{aligned}
$$

В пределе точной $S U(3)_{F}$-симметрии все введенные выше функции имеют определенные свойства симметрии: $\widetilde{\psi}_{2}(\omega, u), \widetilde{\psi}_{3 s}(\omega, u)$ и $\widetilde{\psi}_{4}(\omega, u)$ симметричны по отношению к замене $u \leftrightarrow 1-u$, а $\widetilde{\psi}_{3 \sigma}(\omega, u)$ антисимметрична. Как следствие, только четные полиномы Гегенбауэра входят в симметричные модельные функции для АР, т. е. $a_{1}^{(t)}=0, t=1,2,3$, и нечетные полиномы - в антисимметричную модельную функцию $\left(b_{0}^{(3)}=b_{2}^{(3)}=0\right)$. Если ограничиться только изоспиновой симметрией, то свойства симметрии сохраняются для $\Lambda_{Q}$-бариона, но не для $\Xi_{Q}$, так как вклад $s$-кварка изменится.

Детальный численный анализ барионов с $j^{p}=1^{+}$дикварком будет представлен в работе [8]. На рис. 4 проиллюстрированы функциональные зависимости амплитуд распределения для $J^{P}=1 / 2^{+}$барионов. Неопределенности в АР главным образом определяются произволом $(0 \leqslant A \leqslant 1)$ в выборе локального интерполяционного тока. 
ТАБЛИЦА 4

\begin{tabular}{c|c|ccccc}
\hline$H_{Q}$ & $t$ & $\varepsilon_{0}^{(t)}$ & $\varepsilon_{1}^{(t)}$ & $\varepsilon_{2}^{(t)}$ & $a_{1}^{(t)}$ & $a_{2}^{(t)}$ \\
\hline & 2 & $0.201_{-0.059}^{+0.143}$ & 0 & $0.551_{-0.356}^{+\infty}$ & 0 & $0.391_{-0.279}^{+0.279}$ \\
$\Lambda_{b}$ & 3 & $0.232_{-0.056}^{+0.047}$ & 0 & $0.055_{-0.020}^{+0.010}$ & 0 & $-0.161_{-0.207}^{+0.108}$ \\
& 4 & $0.352_{-0.083}^{+0.067}$ & 0 & $0.262_{-0.132}^{+0.116}$ & 0 & $-0.541_{-0.090}^{+0.173}$ \\
\hline & 2 & $0.228_{-0.061}^{+0.068}$ & $0.429_{-0.281}^{+0.654}$ & $0.449_{-0.473}^{+\infty}$ & $0.057_{-0.034}^{+0.055}$ & $0.449_{-0.380}^{+0.236}$ \\
$\Xi_{b}$ & 3 & $0.258_{-0.038}^{+0.031}$ & $0.750_{-0.093}^{+0.308}$ & $0.520_{-0.060}^{+0.229}$ & $0.339_{-0.160}^{+0.261}$ & $5.244_{-1.132}^{+0.696}$ \\
& 4 & $0.378_{-0.080}^{+0.065}$ & $2.291_{-0.842}^{+\infty}$ & $0.286_{-0.150}^{+0.130}$ & $0.039_{-0.018}^{+0.030}$ & $-0.090_{-0.021}^{+0.037}$ \\
\hline \hline$H_{Q}$ & $t$ & $\eta_{1}^{(t)}$ & $\eta_{2}^{(t)}$ & $\eta_{3}^{(t)}$ & $b_{2}^{(t)}$ & $b_{3}^{(t)}$ \\
\hline$\Lambda_{b}$ & 3 & $0.324_{-0.026}^{+0.054}$ & 0 & $0.633_{-0.0 ? ?}^{+0.0 ? ?}$ & 0 & $-0.240_{-0.147}^{+0.240}$ \\
$\Xi_{b}$ & 3 & $0.218_{-0.047}^{+0.043}$ & $0.877_{-0.152}^{+0.820}$ & $0.049_{-0.012}^{+0.005}$ & $0.037_{-0.019}^{+0.032}$ & $-0.027_{-0.027}^{+0.016}$ \\
\hline
\end{tabular}

\section{6. ВЫВОДЫ}

В рамках КХД в пределе симметрии тяжелых кварков построен полный набор нелокальных операторов струнного типа для тяжелых барионов, находящихся в основном состоянии, со спин-четностями $J^{P}=1 / 2^{+}$и $J^{P}=3 / 2^{+}$. Матричные элементы этих операторов между состоянием тяжелого бариона и вакуумом определяют амплитуды распределения разного твиста в зависимости от тока дикварка. Несколько первых моментов АР вычисляются методом правил сумм КХД с использованием техники нелокальных конденсатов легких кварков. Предложены простые теоретические модели для АР, и их параметры вычислены методом подгонки на основе значений, полученных из правил сумм КХД. Эффекты нарушения $S U(3)_{F}$-симметрии приводят к поправкам порядка $10 \%$.

Благодарности. А.Я. Пархоменко выражает искреннюю признательность за радушный прием организаторам XVI Международного семинара по физике высоких энергий “Quarks-2010”, , а также теоретической группе DESY, где была выполнена основная часть работы, за гостеприимство. Исследование проведено в рамках реализации ФЦП "Научные и научно-педагогические кадры инновационной России" на 2009-2013 гг. (Госконтракт № П2323), при частичной финансовой поддержке Министерства образования и науки РФ по программе "Развитие научного потенциала высшей школы" (проект № 2.1.1/13011) и РФФИ (грант № 11-02-00394-а), а также при частичной финансовой поддержке Министерства образования и науки РФ по программе "Научные и научно-педагогические кадры инновационной России" на 2009-2013 гг. (Госконтракт № П795). 


\section{Список литературы}

[1] X.-G. He, T. Li, X.-Q. Li, Y.-M. Wang, Phys. Rev. D, 74:3 (2006), 034026, 12 pp., arXiv: hep-ph/0606025.

[2] A. Khodjamirian, T. Mannel, N. Offen, Phys. Rev. D, 75:5 (2007), 054013, 18 pp., arXiv: hep-ph/0611193.

[3] F. De Fazio, T. Feldmann, T. Hurth, Nucl. Phys. B, 733:1-2 (2006), 1-30, arXiv: hep-ph/0504088; Erratum 800:1-2 (2008), 405-405.

[4] F. De Fazio, T. Feldmann, T. Hurth, JHEP, 02 (2008), 031, 30 pp., arXiv: 0711.3999.

[5] W. Loinaz, R. Akhoury, Phys. Rev. D, 53:3 (1996), 1416-1424, arXiv: hep-ph/9505378.

[6] F. Hussain, J. G. Körner, M. Krämer, G. Thompson, Z. Phys. C, 51:2 (1991), 321-327.

[7] P. Ball, V. M. Braun, E. Gardi, Phys. Lett. B, 665:4 (2008), 197-204, arXiv: 0804.2424.

[8] A. Ali, C. Hambrock, A. Parkhomenko, LCDAs of ground-state bottom baryons (in preparation).

[9] A. G. Grozin, Heavy Quark Effective Theory, Springer Tracts in Modern Physics, 201, Springer, Berlin, Heidelberg, 2004.

[10] A. G. Grozin, O. I. Yakovlev, Phys. Lett. B, 285:3 (1992), 254-262, arXiv: hep-ph/9908364.

[11] S. Groote, J. G. Körner, O. I. Yakovlev, Phys. Rev. D, 56:7 (1997), 3943-3954, arXiv: hep-ph/9705447.

[12] E. V. Shuryak, Nucl. Phys. B, 198:1 (1982), 83-101.

[13] Y.-B. Dai, C.-S. Huang, C. Liu, C.-D. Lü, Phys. Lett. B, 371:1-2 (1996), 99-104, arXiv: hep-ph/9602242.

[14] A. F. Falk, Nucl. Phys. B, 378:1-2 (1992), 79-94.

[15] P. Ball, V. M. Braun, Y. Koike, K. Tanaka, Nucl. Phys. B, 529:1-2 (1998), 323-382, arXiv: hep-ph/9802299.

[16] E. Bagan, M. Chabab, H. G. Dosch, S. Narison, Phys. Lett. B, 301:1-2 (1993), 243-248.

[17] S. Groote, J. G. Körner, O. I. Yakovlev, Phys. Rev. D, 55:5 (1997), 3016-3026, arXiv: hep-ph/9609469.

[18] B. O. Lange, M. Neubert, Phys. Rev. Lett., 91:10 (2003), 102001, 4 pp., arXiv: hep-ph/0303082.

[19] G. P. Lepage, S. J. Brodsky, Phys. Lett. B, 87:4 (1979), 359-365; Phys. Rev. D, 22:9 (1980), 2157-2198; А. В. Ефремов, А. В. Радюшкин, ТМФ, 42:2 (1980), 147-166; A. V. Efremov, A. V. Radyushkin, Phys. Lett. B, 94:2 (1980), 245-250.

[20] A. G. Grozin, M. Neubert, Phys. Rev. D, 55:1 (1997), 272-290, arXiv: hep-ph/9607366.

[21] V. M. Braun, D. Y. Ivanov, G. P. Korchemsky, Phys. Rev. D, 69:3 (2004), 034014, 11 pp., arXiv: hep-ph/0309330.

[22] С. В. Михайлов, А. В. Радюшкин, Писъма в ЖЭТФ, 43:12 (1986), 551-553.

[23] S. V. Mikhailov, A. V. Radyushkin, Phys. Rev. D, 45:5 (1992), 1754-1759.

[24] V. Braun, P. Górnicki, L. Mankiewicz, Phys. Rev. D, 51:11 (1995), 6036-6051, arXiv: hep-ph/9410318.

[25] A. G. Grozin, Int. J. Mod. Phys. A, 10:24 (1995), 3497-3529, arXiv: hep-ph/9412238.

[26] K. Nakamura et al. (Particle Data Group), J. Phys. G, 37:7A (2010), 075021, 1422 pp.

[27] X. Liu, H.-X. Chen, Y.-R. Liu, A. Hosaka, S.-L. Zhu, Phys. Rev. D, 77:1 (2008), 014031, 8 pp., arXiv: 0710.0123

[28] R. Lewis, R. M. Woloshyn, Phys. Rev. D, 79:1 (2009), 014502, 9 pp., arXiv: 0806.4783.

[29] J.-R. Zhang, M.-Q. Huang, Phys. Rev. D, 78:9 (2008), 094015, 12 pp., arXiv: 0811.3266.

[30] K. G. Chetyrkin, A. Khodjamirian, A. A. Pivovarov, Phys. Lett. B, 661:4 (2008), 250-258, arXiv: 0712.2999.

[31] V. M. Braun, I. E. Filyanov, Z. Phys. C, 44:1 (1989), 157-166; В. М. Браун, И. Е. Филянов, ЯФ, 50:3 (1989), 818-830.

[32] V. M. Braun, I. E. Filyanov, Z. Phys. C, 48:2 (1990), 239-247; В. М. Браун, И. Е. Филянов, ЯФ, 52:1 (1990), 199-213.

[33] P. Ball, JHEP, 01 (1999), 010, 19 pp., arXiv: hep-ph/9812375. 